\title{
Prophylactic Effects of Silver Nanoparticles In A Rat Model Of Spinal Stabilization and Infection
}

\author{
Abdurrahim Tas ${ }^{1 *}$, Feyza Karagoz Guzey ${ }^{2}$, Azmi Tufan ${ }^{2}$, Nuray Kuvat ${ }^{3}$, Özgür Yusuf \\ Aktaş $^{4}$, Murat Yücel ${ }^{5}$, Asli Kahraman Akkalp ${ }^{6}$ \\ ${ }^{1} Y$ uzuncu Yal University, School of Medicine Department of Neurosurgery Van, Turkey \\ ${ }^{2}$ Health Sciences University Bağcılar Training Research Hospital Neurosurgery Clinu Istanbul,Turkey \\ ${ }^{3}$ Abenk laboratory Clinical Microbiology Clinic İstanbul,Turkey \\ ${ }^{4}$ Manisa city hospital Neurosurgery Clinc Manisa,Turkey \\ 5 Sivas Numune Hospital Neurosurgery Clinc Sivas, Turkey \\ ${ }^{6}$ Katip Celebi University Ataturk. Training and Research Hospital, Department of Pathology Izmir, Turkey
}

\begin{abstract}
Several factors increase the risk of postoperative infection. Some experimental studies have shown that silver nanoparticles (AgNP) have a protective effect from surgical infection. This study aimed to determine the effectiveness of AgNP in preventing the development of infection in the spinal stabilization rat model.

In this study, 32 Sprague-Dawley rats weighing 300-350 g were used. Subjects were randomly divided into four groups. Titanium screws were placed in the spine in all groups. In Groups 2, 3, and 4, S.aureus and P.aeruginosa were cultivated in the surgical site at a concentration of $106 \mathrm{CFU} / 10 \mu \mathrm{L}$. Additionally, $0.5 \mathrm{mg} / \mathrm{kg}$ AgNP in maltose solution in Group 3 and maltose solution in Group 4 were used. The histopathological examinations were then done.

In the fascia, muscle, bone, and screws in all groups, except in Group 1 (control group), S. aureus and P.aeruginosa reproduced. In blood cultures, there was growth of S. aureus and P. aeruginosa in 1 subject in Group 2, 6 subjects in Group 3, and 2 subjects in Group 4. The growth of S. aureus and P.aeruginosa was significantly increased in Group 3. The degree of inflammation was significantly lower in Group 1. No accumulation of silver was detected in any organs histopathologically.

Application of 1 eucal $\mathrm{AgNP}$ decreased the severity of infection. This dose was not toxic. However, administration of $\mathrm{AgNP}$ at this dose did not decrease the local infection rate. Therefore, new studies should be conducted to determine the ideal effective dose.
\end{abstract}

Keywords: Rat, surgical infection, silver nanoparticle, spinal stabilization

\section{Introduction}

The surgical infection rate in spinal surgery procedures without instrumentation, such as discectomy and laminectomy, is up to $1 \%(1,2)$. The presence of dead space in the surgical field, foreign bodies, necrotic tissues that have lost their vitality, and long-term surgical interventions increases the risk of postoperative infection by disrupting the host defense (3). Adding instrumentation in spinal surgery increases the surgical infection rate to $2.1-8.5 \%(1,4,5)$.

Surgical infection prolongs patient's length of hospital stay and increases morbidity and mortality $(1,3,6)$. When treatment cost is calculated, a patient who develops osteomyelitis is at least four times more costly than a patient who has only stabilization. Since some patients are unable to return to their previous jobs, their indirect costs actually reach much larger levels $(1,7)$.

After the instruments are implanted into the host tissue, a biofilm layer with a glycoprotein structure is formed on their surface containing fibronectin, fibrinogen, collagen, and other proteins. This layer forms a receptor site necessary for the adhesion of bacteria and host tissue cells $(1,3)$. Microcolonies that develop in the biofilm layer adhering to the implant surface are protected against both antimicrobial therapy and host defense $(8,9)$. For this reason, implant-related infections are less likely to respond to antibiotics and cannot be often controlled until the implant is removed $(1,8,9)$. Therefore, antimicrobial prophylaxis is greatly important in implant interventions.

${ }^{*}$ Corresponding Author: Abdurrahim Tas, Van Yuzuncu Y1l University, School of Medicine Department of Neurosurgery Van, Turkey E-mail: drabdurrahimtas@gmail.com

ORCID ID: Abdurrahim Tas: 0000-0001-5786-9063, Feyza Karagoz Guzey: 0000-0002-4260-9821, Azmi Tufan: 0000-0001-9042-8542, Nuray Kuvat: 0000-0003-3979-6475, Özgür Yusuf Aktaş: 0000-0001-8826-4139, Murat Yücel: 0000-0002-0826-5965, Asli Kahraman Akkalp: 0000-0001-5781-8506

Received: 02.08.2021, Accepted: 15.12.2021 
This study aimed to determine the effectiveness of using local silver nanoparticle (AgNP) as an alternative treatment modality in preventing spinal infection developing after instrumentation in rat spine.

\section{Materialsand Methods}

Preparation of Bacteria and Inoculate: In our study, Staphylococcus aureus and Pseudomonas aeruginosa, which were among the most common factors in implant-related infections in our hospital, were used (Becton Dickinson, Staphylococcus aureus ATCC 29213 and Pseudomonas aeruginosa ATCC 27853). Strains were added to tryptic soy broth after sterilization in water from salt, diluted with two layers, and centrifuged at $3000 \mathrm{rpm}$ for $10 \mathrm{~min}$. Bacterial sediment was added to phosphate buffered water until McFarland 6 standard was achieved. Dilutions containing the desired number of bacteria were prepared and transferred to the freezer at $-70^{\circ} \mathrm{C}$. When applied to rats, it was removed from the freezer, and 15 micron (106 colony-forming units $[\mathrm{CFU}] / 10 \mu \mathrm{L})$ bacterial dilution was applied to each subject (10).

Preparation Of Subjects and Surgical Intervention: The study was conducted at Bağcilar Training and Research Hospital Experimental Research and Skill Development Center after obtaining approval from the local ethics committee $\left(47^{\text {th }}\right.$ Board meeting decision of the project number 24 of 2015, dated 06.08.2015). In the study, 32 4-6-month-old male SpragueDawley rats weighing 300-350 g were used. Subjects were randomly numbered, and four groups with repairs were created:

$>$ Group 1 (negative control group): 3-mm microscrew $(\mathrm{n}=8)$

$>$ Group 2 (control group): 3-mm microscrew $+\mathrm{S}$. aureus and P. aeruginosa $106 \mathrm{CFU} / 10 \mu \mathrm{L}(\mathrm{n}=8)$

$>$ Group 3: 3-mm microscrew + maltose solution (solvent for AgNP) $+\mathrm{S}$. aureus and P. aeruginosa $106 \mathrm{CFU} / 10 \mu \mathrm{L}(\mathrm{n}=8)$

$>$ Group 4: 3-mm microscrew + AgNP + S. aureus and $\mathrm{P}$. aeruginosa $106 \mathrm{CFU} / 10 \mu \mathrm{L}(\mathrm{n}=8)$

Subjects were administered $7 \mathrm{mg} / \mathrm{kg}$ xylazine (Rompun 2\% $25 \mathrm{~mL}$, Bayer) and $80 \mathrm{mg} / \mathrm{kg}$ ketamine (Ketaral $500 \mathrm{mg}$, Pfizer) i.m. General anesthesia was performed with vertical skinsubcutaneous incision in the midline of $1-1.5 \mathrm{~cm}$ length at T10-12 level. The paravertebral fascia was opened, and the muscles were unilaterally removed from the spinous process and lamina. A part of the lamina was decorticated. The screw entry slot was opened with the number 11 scalpel tip, and a 3-mm-long titanium microscrew was inserted and tightened with a screwdriver (11).

In Group 1, a 3-mm titanium microscrew was applied to the pedicle as a negative control group. In Group 2, 3 and 4, after applying the microscrew to the pedicle as a control group, bacteria dilution ( $S$. aureus and $P$. aeruginosa) in a volume of $106 / 10 \mu \mathrm{L}$ was dropped on the screw using a $50 \mu \mathrm{L}$ micropipette. In Group 3, operation field and screw was washed with a solvent solution $(1 \mathrm{~mL})$ consisting of maltose and water without nanoparticles. In Group 4, operation field and screw was washed with $1 \mathrm{~mL}(0.5 \mathrm{mg} / \mathrm{kg})$ AgNP solution (Nanoclearag, Istanbul, Turkey) and dried.

Obtaining Microbiological Samples and Scarification: After 15 days, subjects were anesthesized with $7 \mathrm{mg} / \mathrm{kg}$ xylazine $+80 \mathrm{mg} / \mathrm{kg}$ ketamine i.m. Infection findings in the surgical field were clinically evaluated. Then, after obtaining $3 \mathrm{~mL}$ of intracardiac blood culture, the subjects were sacrificed by applying a deadly dose of $10 \mathrm{mg}$ potassium chloride.

In the sterile surgical environment, the skin was opened under the old incision site. Samples were obtained from the fascia and muscle. The screw was removed with a screwdriver and placed in a sterile culture tube. Different sterile instruments were used in each tissue when obtaining microbiological samples. Obtained samples were placed in $15 \mathrm{~mL}$ sterile Falcon tubes and sent to the microbiology laboratory for cultivation. Moreover, samples were obtained from the vertebra for histological evaluation from materials containing bone and surrounding soft tissue. Brain, liver and kidney tisuues were obtained for histopathological evaluation also.

Blood from the subjects was transferred to BD Bactec Plus Aerobic/F blood culture bottles. The Bactec FX (Becton Dickinson, USA) automated blood culture device was used for incubation in the laboratory. Passage cultivation was performed in those who had reproduction warning from culture bottles followed for seven days. Growing bacteria were identified using the Phoenix (Becton Dickinson, USA) automated device.

All microbiological samples were subjected to tryptic strain. After $24 \mathrm{~h}$ of incubation at $37^{\circ} \mathrm{C}$, blood agar and chromogenic UTI agar media were sown. Reproduction in these media was assessed after 24 h. Growing bacteria were identified using the Phoenix (Becton Dickinson, USA) automated device. 
Histopathological Evaluation: After the vertebral specimens of all groups, brain, liver and kidney tissues were detected with 10\% neutral formalin, the vertebral specimens were also decalcified in $10 \%$ nitric acid solution. When the cuttable softness was reached, all tissues were sliced transverse perpendicular to the vertebral column axis. Paraffin blocks were prepared for all samples; then, 4-mu-thick sections were prepared and stained with hematoxylin-eosin (H\&E) dye.

All sections were evaluated and scored by the same pathologist using a light microscope based on severity of inflammation as follows: minimal $(0)$, mild $(+)$, moderate $(++)$, and severe $(+++)$ (10).

Samples obtained from the brain, lung, liver, and kidney were evaluated in H\&E-stained preparations for inflammatory foreign body reactions that may occur in the presence of silver accumulation in the light microscope.

Statistical Analysis: Statistical analyzes were performed with the NCSS (Number Cruncher Statistical System) 2007 Statistical Software (Utah, USA) package program.

In the evaluation of the data; In addition to descriptive statistical methods (frequency and percentage distributions), Fisher Exact Chi-Square test were used for comparison of qualitative data. The results were evaluated at the significance level of $\mathrm{p}<0.05$.

\section{Results}

Clinical Evaluation of Infection: In all bacteriacultivated groups (Groups 2, 3 and 4), infection in the skin, subcutaneous fascia, and bone was macroscopically observed. Skin and subcutaneous abscess and S. aureus and P. aeruginosa pigments were noted. Infection findings were most prominent in Group 3, in which pus was overflowing from the skin. After the skin and subcutaneous fascia were opened, yellow purulent material was observed in all rats in Groups 2, 3, and 4, with more purulent material in Group 3.

Microbiological Findings: S. aureus and P. aeruginosa were grown in cultures from the fascia, muscle, bone, and screws in all groups (Groups 2, 3 , and 4) in which bacteria were cultivated. In blood cultures, there was growth of P. aeruginosa and S. aureus in $1(12.5 \%)$ subject in Group 2, 6 $(75 \%)$ subjects in Group 3, and $2(25 \%)$ subjects in Group $4(\mathrm{p}=0.005)$. When analyzed among themselves, a statistically significant difference was found between group 3 with groups 1, 2, 4 $(\mathrm{p}=0.002,0.008$, and 0.046 , respectively).The reproduction status in the cultures obtained from the subjects in all groups is shown in Table 1.

\section{Histopathological}

Findings: Histopathologically, inflammation was detected in $1(12.5 \%)$ subject in group 1 , in $5(62.5 \%)$ subjects in group 2, and in all subjects in groups 3 and 4 $(p=0.007)$.In the analysis of the groups according to the presence of inflammation, a statistically significant difference was found between group 1 and groups 2, 3, $4 \quad(p=0.045,0.001,0.003$, respectively). The degree of inflammation histologically detected at the operative site in all groups is presented in Table 2, and examples of histological views of different degrees of inflammation are shown in Figures $1 \mathrm{a}$ and b. No accumulation of silver was observed in the brain, liver, kidney, and lung tissues in any subjects (Figure 2).

\section{Discussion}

Surgical infection is a severe problem after spinal operations, especially instrumentation, and a serious cause of morbidity and sometimes mortality. Systemic or local drugs and substances have been used in human and animal studies in the prevention of these infections. These include systemically applied antibiotics and locally applied vancomycin, antibiotic-added fibrin tissue adhesive, betadine, royal jelly, Manuka honey, and Propolis (10). For example, various implants impregnated with antibiotics have been produced and started to be used (12). A recent prospective clinical study by Eren et al. reported that immersion of the instrument in vancomycin and ceftriaxone solution before administration reduced the risk of deep infection (1). However, since no fully effective and safe agent is found, both clinical and experimental studies are still ongoing.

It is known that silver compounds and ions are cytotoxic for a long period and used in the eradication of microorganisms. Silver is used in a wide range of commercial products and medical devices due to its relatively low toxicity. According to the American Environmental Protection Agency guidelines, the maximum silver rate in drinking water is $100 \mathrm{ppb}$. Additionally, additives containing AgNP are contemplated for use in pet food, but the effect of AgNP or its derivatives in controlling microbial infections in the animal's digestive system has not yet been confirmed (13). 
Tas et al / İnfection, effects of silver particles in rats

Table 1. Microorganism Growth In Cultures From Subjects (There Are 8 Subjects In Each Group)

\begin{tabular}{lccccc}
\hline Locations & Group 1, n (\%) & Group 2, n (\%) & Group 3, n (\%) & Group 4, n (\%) & p values \\
\hline Fascia & $0(0)$ & $8(100)$ & $8(100)$ & $8(100)$ & $<0.001$ \\
Muscle & $0(0)$ & $8(100)$ & $8(100)$ & $8(100)$ & $<0.001$ \\
Bone & $0(0)$ & $8(100)$ & $8(100)$ & $8(100)$ & $<0.001$ \\
Screw & $0(0)$ & $8(100)$ & $8(100)$ & $8(100)$ & $<0.001$ \\
Blood & $0(0)$ & $1(12.5)^{*}$ & $6(75)$ & $2(25)$ & 0.005 \\
\hline
\end{tabular}

*only Pseudomonas aeruginosa positive; in other positive cultures both microorganisms were yielded

Table 2. Inflammation Degree (There Are 8 Subjects In Each Group)

\begin{tabular}{lccccc}
\hline Inflammation & Group 1, n (\%) & Group 2, n (\%) & Group 3, n (\%) & Group 4, n (\%) & p value \\
\hline No & $7(87.5)$ & $3(37.5)$ & $0(0)$ & $0(0)$ & \\
Yes & & & & & \\
Mild & $1(12.5)$ & $0(0)$ & $0(0)$ & $1(12.5)$ & 0.007 \\
Moderate & $0(0)$ & $3(37.5)$ & $5(62.5)$ & $4(50)$ & \\
Severe & $0(0)$ & $2(25)$ & $3(37.5)$ & $3(37.5)$ & \\
\hline
\end{tabular}

With the recent development of nanoparticle technology, binding different substances to nanoparticles and using them especially in local treatments have become widespread. Soluble silver ion species and colloidal AgNP are known to be good antiseptics against broad-spectrum microorganisms and antibiotic-resistant bacteria $(14,15)$. In addition, in some studies, AgNP has been shown to be superior in inhibiting bacterial growth compared to traditional silver ions $(16,17)$.

In our study, local AgNP application did not prevent local infection and systemic spreading of infection according to the control group without any treatment (Group 2). However, we performed the AgNP in a maltose solution as solvent because of its commercial product, and maltose solution is a nourishing media for microorganisms. Indeed, in Group 3 in which only maltose solution was performed without AgNP, macroscopic findings of the infection with purulent drainage from the skin over the operation site, and microscopic inflammation findings were apparent according to the control groups, and positive blood culture rate also was very high. However, AgNP addition to the maltose solution provided to decrease positive blood culture rate to the similar degree with Group 2, and to lessen the macroscopic findings of infection. These results revealed that AgNP applied locally at a dose of $0.5 \mathrm{mg} / \mathrm{kg}$ prevents systemic spreading of the local surgical infection, and also provides to lessen the degree of local infection. If another solvent solution that cannot be used as a nourishing media by the microorganisms is chosen, AgNP may prevent the development of local infection also.
In the literature, similar studies reported that AgNP at $0.25 \mathrm{mg} / \mathrm{kg}$ dose has $25 \%$ local infection control at $1 \mathrm{mg} / \mathrm{kg}$ dose $(16,18,19)$. This data contradicts the result of our study. This may be due to the number of inoculated bacteria. We performed the standard $106 \mathrm{CFU} / 10 \mu \mathrm{L}$ bacterial inoculation, whereas other studies performed 100 $\mathrm{CFU} / \mathrm{mL}$ bacterial inoculations. At this point, bacterial inoculation amount gains importance in the effect of AgNP on local infection control. Moreover, two different strains were used together for infection model. This may be effective in reducing the effectiveness of $\mathrm{AgNP}$. Nevertheless, we want to simulate our surgical theatre. These two microorganisms are the most frequent agents causing surgical infection after instrumentation in our hospital.

Additionally, cytotoxicity and accumulation of $\mathrm{Ag}$ deposits in tissues are important safety concerns (20). The main organ in which silver accumulates is the liver, followed by the spleen, lungs, and kidneys (18). Silver causes oxidative stress and formation of reactive oxygen products in cell membrane and all organelles, including lysosome, mitochondria, and nucleus, resulting in necrosis or apoptosis. Considering the relative toxicities of AgNP and Ag ions medically, AgNP has been shown to be three times less toxic to human monocytes and zebrafish than $\mathrm{Ag}$ ions $(21,22)$. Oxidative stress caused by AgNP can trigger inflammatory response with natural immune activation and increased permeability in endothelial cells. AgNP can also cause deoxyribonucleic acid (DNA) damage, 

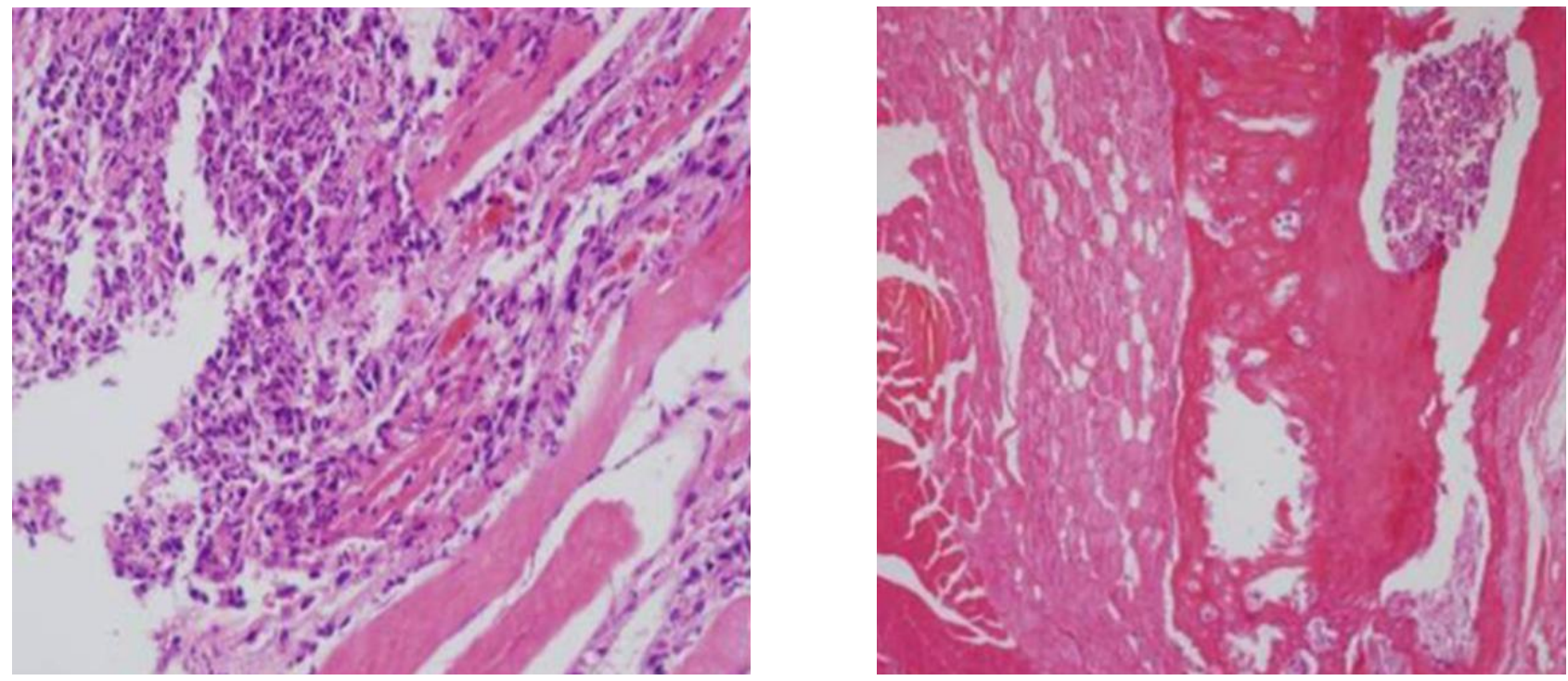

Fig. 1. a) Inflammation (+++) (Group 4) (H\&E, 40×). b) Inflammation: (++), pronounced congestion (Group 3$)$ (H\&E, 10×).
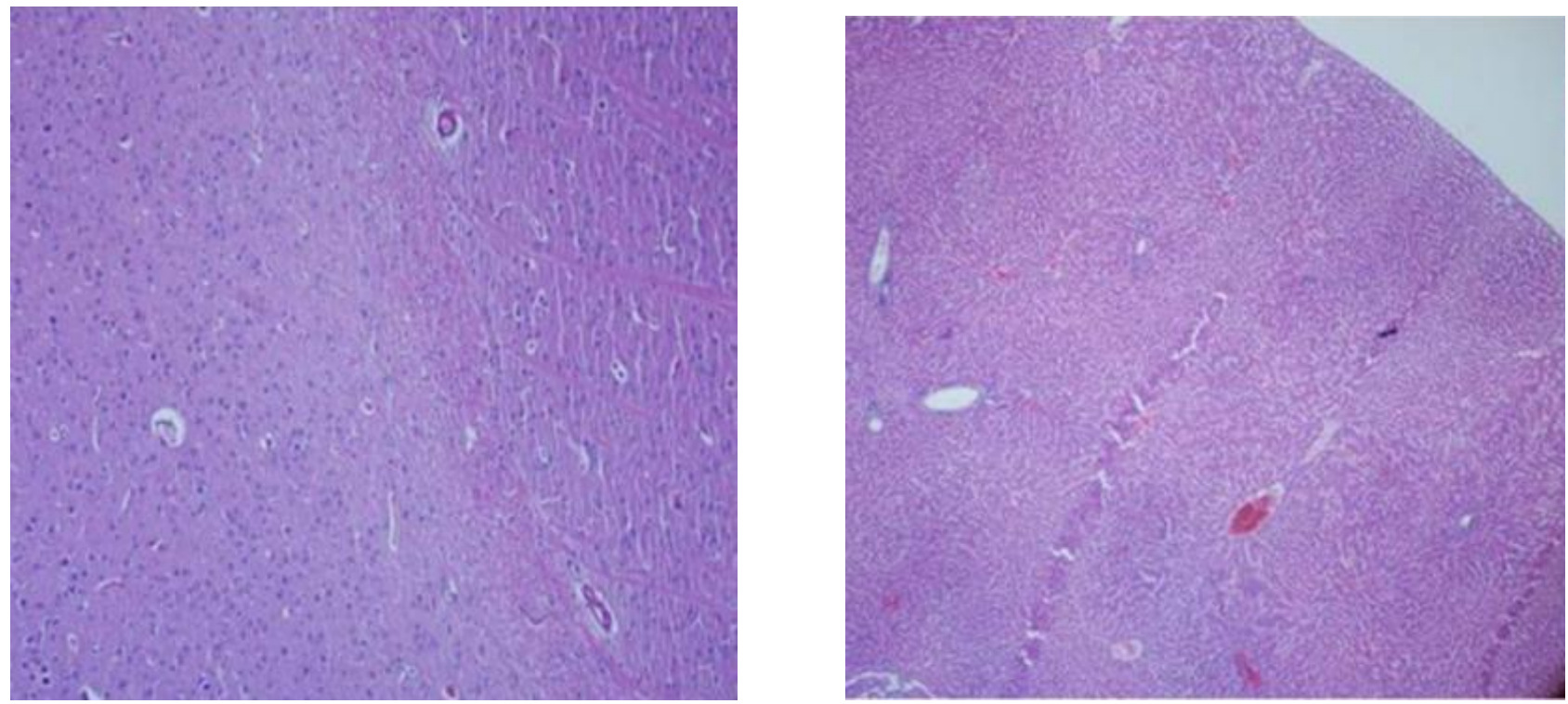

A

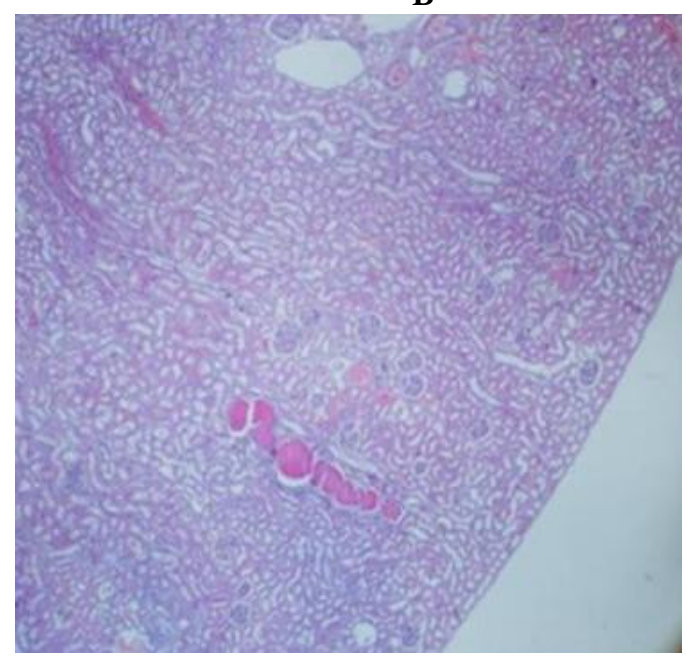

C

Fig. 2. Brain (a), liver (b), and kidney (c) tissues without silver deposition

East J Med Volume:27, Number:1, January-March/2022 
Tas et al / Infection, effects of silver particles in rats

chromosomal anomaly, and possible gene mutation, even if not at the cytotoxic dose (18).

In our study, we observed that silver did not accumulate in the liver, lung, kidney, and brain at $0.5 \mathrm{mg} / \mathrm{kg}$ local administration. However, AgNP at this dose was insufficient in local infection control. Therefore, new studies are needed to determine the ideal effective dose in prophylactic administration of local AgNP, which will also be effective in preventing local infection but will not lead to silver accumulation in the tissues.

Silver nanooparticles, which was administered at $0.5 \mathrm{mg} / \mathrm{kg}$ with local infection, had no significant side effect in inoculated groups but was observed to be significantly effective in terms of preventing sepsis. Although this dose did not prevent local infection, decreased sepsis rates suggested that local application of AgNP decreased the severity of infection. AgNP accumulation was not found in the other organs. It seemed that the selected dose was toxic to microorganisms, but insufficient to prevent local infection. New studies are required on the ideal effective dose for the local application of AgNP. Also, more inert solvents than maltose must be evaluated.

Infections are one of the most important factors affecting mortality and morbidity in patients with lumbar stabilization. New approaches are being developed every day for infection prophylaxis in patients undergoing lumbar stabilization.Silver nanoparticles used prophylactically in rats with vertebral implants reduce the severity of infection.

Acknowlegement: Thanks to Biostatistic consultan Dr. Rana.

Financial Support: This research work was funded by Scientific Research Project Coordination Center of Bağcllar Training and Research Hospital (Project Number: 2015-24).

Conflict of interest: None declared.

Ethical Approval: Bağcılar Ttraining and Research Hospital Animal Experiments Local Ethics Committee Decision: 47th Board meeting decision of the project number 24 of 2015, dated 06.08.2015

\section{References}

1. Eren B, Karagöz Güzey F, Kitiş S, Özkan N, Korkut C. The effectiveness of pedicle screw immersion in vancomycin and ceftriaxone solution for the prevention of postoperative spinal infection: A prospective comparative study. Acta Orthop Traumatol Turc 2018; 52: 289-293.
2. Horwitz NH, Curtin JA. Prophylactic antibiotics and wound infections following laminectomy for lumbar disc herniation. A retrospective study. J Neurosurg 1975; 43: 727-731.

3. Tofuku K, Koga H, Yanase M, et al. The use of antibiotic-impregnated fibrin sealant for the prevention of surgical site infection associated with spinalinstrumentation. Eur Spine J 2012; 21: 2027-2033

4. O'Toole JE, Eichholz KM, Fessler RG. Surgical site infection rates after minimallyinvasive spinal surgery. J Neurosurg Spine 2009; 11: 471-476.

5. Khan NR, Thompson CJ, DeCuypere M, et al. A meta-analysis of spinal surgicalsite infection and vancomycin powder. J Neurosurg Spine 2014; 21: 974-983.

6. Chang FY, Chang MC, Wang ST, Yu WK, Liu $\mathrm{CH}$, Chen TH. Can povidone-iodine solution be usedsafely in a spinal surgery? Eur Spine J 2006; 15: 1005-1014.

7. Ahmed R, Greenlee JD, Traynelis VC. Preservation of spinal instrumentationafter development of postoperative bacterial infections in patients undergoingspinal arthrodesis. J Spinal Disord Techn 2012; 25: 299-302.

8. Secinti KD, Ayten M, Kahilogullari G, et al. Antibacterial effects of electricallyactivated vertebral implants. J Clin Neurosci 2008; 15: 434-439.

9. Zhang T, Wang L, Chen Q, et al. Cytotoxic potential of silver nanoparticles.Yonsei Med J 2014; 55: 283-291.

10. Gunald1 O, Daglıoglu YK, Tugcu B, et al. Antibacterial Effect of Royal Jelly for Preservation of Implant-Related Spinal Infection in Rat. Turk Neurosurg 2014; 24 : 249-252.

11. Ofluoglu EA, Zileli M, Aydin D, et al. Implant-related infection model in rat spine. Arch Orthop Trauma Surg 2007; 127: 391-396.

12. Greene AH, Bumgardner JD, Yang Y, Moseley J, Haggard WO. Chitosan-coated stainlesssteel screws for fixation in contaminated fractures. Clin. Orthop Relat Res 2008; 4667: 1699-1704.

13. Fondevila M, Herrer R, Casallas MC, Abecia L, Ducha JJ. Silver nanoparticles as a potential antimicrobial additive for weaned pigs. Anim Feed Sci Technol 2009; 150:259-269.

14. Jung WK, Koo HC, Kim KW, Shin S, Kim SH, Park YH. Antibacterial activity and mechanism of action of the silver ion in Staphylococcus aureus and Escherichia coli. Appl Environ Microbiol 2008; 74: 2171-2178.

15. Shahverdi AR, Fakhimi A, Shahverdi HR, Minaian S. Synthesis and effect of silver nanoparticles on the antibacterial activity of

East J Med Volume:27, Number:1, January-March/2022 
different antibiotics against Staphylococcus aureus and Escherichia coli. Nanomedicine 2007; 3: 168-171.

16. Choi O, Deng KK, Kim NJ, Ross L Jr, Surampalli RY, Hu Z. The inhibitory effects of silver nanoparticles, silver ions, and silver chloride colloids on microbial growth. Water Res 2008; 42: 3066-3074.

17. Su HL, Lin SH, Wei JC, et al. Novel nanohybrids of silver particles on clay platelets for inhibiting silver-resistant bacteria. PLoS One 2011; 6: e21125.

18. Lankveld DP, Oomen AG, Krystek P, et al. The kinetics of the tissue distribution of silver nanoparticles of different sizes. Biomaterials 2010; 31: 8350-8361.

19. Lall RR, Wong AP, Lall RR, Lawton CD, Smith ZA, Dahdaleh NS. Evidence-based management of deep woundinfection after spinal instrumentation. J Clin Neurosci 2015; 22: $238-242$.

20. Chaloupka K, Malam Y, Seifalian AM. Nanosilver as a new generation of nanoproduct in biomedical applications. Trends Biotechnol 2010; 28: 580-588.

21. Foldbjerg R, Olesen P, Hougaard M, Dang DA, Hoffmann HJ, Autrup H. PVP-coated silver nanoparticles and silver ions induce reactive oxygen species, apoptosis and necrosis in THP-1 monocytes. Toxicol Lett 2009; 190: 156-162.

22. Bilberg K, Hovgaard MB, Besenbacher F, Baatrup E. In vivo toxicity of silver nanoparticles and silver ions in zebrafish (Danio rerio). J Toxicol 2012; 293784. 